\title{
Scientific Research Through Simulation in Africa: Role of HPC in Computational Material Studies
}

\author{
Elicah Nafula Wabululu, ${ }^{1}$,, P. W. O. Nyawere ${ }^{2}$, Daniel Barasa Bem ${ }^{1}$ \\ ${ }^{1}$ Department of Physics, Kenyatta University, Nairobi, Kenya \\ ${ }^{2}$ Department of Physical Sciences, Rongo University, Rongo, Kenya
}

Email address:

ellyvinny@gmail.com (E. N. Wabululu)

${ }^{*}$ Corresponding author

\section{To cite this article:}

Elicah Nafula Wabululu, P. W. O. Nyawere, Daniel Barasa Bem. Scientific Research Through Simulation in Africa: Role of HPC in Computational Material Studies. International Journal of Materials Science and Applications. Vol. 6, No. 4, 2017, pp. $190-192$. doi: $10.11648 /$ j.ijmsa.20170604.14

Received: April 21, 2017; Accepted: May 17, 2017; Published: July 6, 2017

\begin{abstract}
Africa is not investing much in computational materials research. Poor infrastructure in terms of electricity and internet connectivity has made research difficult for computational researchers. High Performing Computers and especially CHPC of South Africa have proven to be a better way of doing research in Africa. It has attracted many researchers in Physics, Chemistry and Biology just to name a few. In these initial studies, $\mathrm{LaF}_{3}$ has been presented as a case study currently being done in CHPC yet miles away from Kenya. With the actual speed achieved and resources used (e.g. twelve times speed and over $20 \mathrm{CPUs}$ ). For the lattice parameter for $\mathrm{LaF}_{3}$, the experimental work has given a value of $7.20 \mathrm{Bohr}$ and $7.36 \mathrm{Bohr}$ respectively while our computational results are 7.24 Bohr and 7.37 Bohr respectively. The deviation between experimental and computational proves to be small hence validating our computational research.
\end{abstract}

Keywords: High Performing Computers, Computer Simulations, Lattice Parameter

\section{Introduction}

A country's economic growth can be pegged on the research it does. In western countries, governments have discovered this relationship and have heavily invested in research. In Africa, our challenge still lies on food and a lot of funds are used to either alleviate hunger or fight drought challenges. Apart from South Africa that has discovered this secret of inter-relations between economic growth and research most countries in Africa are struggling with funding scientific research. Through establishment of the Center of High Performing Computers (CHPC) in 2007, South Africa has positioned herself as a hub for research and a preferred destination for computational researchers. It has also enabled the researchers from this facility to contribute to regional and international views in many disciplines $h$ [1]. In Kenya, 2016/2017 budget saw the government allocate Ksh 3 billion which is an improvement for the first time to $2 \%$ of the national budget allocated to research. Most of this fund is disbursed to applied sciences where much emphasis is given to biological science and environmental science [2]. This may be because most of those in the panel tasked with assessing the applicants are not familiar with computational research or we still don't have many experts in the area. The assessors want ordinary data collection research that gives quick fix results. The desire for tangible and immediate results on social and economic issues makes computational research non-attractive for funding by most funding bodies.

From 2009, Kenya has seen many students going for computational research either in Physics or Chemistry out of the country. Most of the students have gone for their studies in South Africa or Europe. Because of brain drain, most of these students don't return home because of good opportunities they are offered in these foreign countries.

In Kenya, the active computational group of university of Eldoret may not handle huge calculations given that it was made for the use by the students and staff of the university. This facility was funded by National Commission for Science, Technology and Innovation (NACOSTI) before the formation of National Research Fund [3]. Computational materials research is convenient for Africa because of the 
access that can be done through internet. Secondly, not much equipment is needed like in experimental Physics to set it up. With one center set up, all one requires is network connectivity. It is initial setting that is resource consuming but the benefits are enormous. One such facility can be used by so many virtual users at ago.

In Physics, some of the trained experts have returned to also train the fellow citizens. Unfortunately, most researchers in Kenya rise to administration in their universities leaving the research to the under qualified. Alternatively, some opt for government appointments in other sectors not necessarily research. Some even opt for politics because of the hefty salaries drawn from these sectors. Politicians earn ten times more than a lecturer in Kenya. With facilities being a problem, many scientists opt out to other sectors including private sectors.

In Africa, computational studies would prove to be the best because of ill prepared laboratories and lack of technical staff to assist in advanced research especially in materials research. Most of the laboratories in our state universities are outdated and may not meet the needs of twenty first century research. With the digital era, equipment needs to be updated to catch up with changing trends.

The other method in materials research is theoretical which is good but due to lack of properly stocked libraries may not yield much in developing countries. Many theoretical researches done today require computational backing and with the many softwares available, it is only wise to route for proper computational work in Africa. With the speed of computers having been improved drastically, high performing computers are tools that are irresistible in this generation.

High-performance systems often use custom-made components in addition to so-called commodity components. This increases speed for calculations as compared to normal desk top computers or laptops. The most common users of High Performance Computing (HPC) systems are scientific researchers, engineers and academic institutions. Some government agencies, particularly the military, also rely on HPC for complex applications [4].

Computational research is able to make African researchers be in the frontier of research and equally compete with those in the rest of the word. Like in Quantum Espresso code and other simulations codes, a platform is available for interactions between the users. This enables members to assist each other and also learn new skills being launched almost daily. Contributions from users aid those still learning creating a platform for interaction.

\section{Computational Methods}

In materials research, the properties of materials can be calculated using specific codes for specific purposes. In Physics and Chemistry, many codes [5] are available such as ABINIT, VASP, QUANTUM ESPRESSO, MATLAB, CASTEP just to mention a few. What has been done in an experimental work can be simulated in computers and properties such as conductivity, band gap, reflectivity, energetic of materials, elastic constants among others can be calculated. The deviation between experimental and computational will be there but in most cases when the software is good the percentage deviation can be as small as $0.3 \%$. These deviations arise in the accuracy of pseudopotentials used in calculating these properties and in some cases the condition at which either of the two is carried out. Most of the computational work is done in assumed room temperature which may not be a true environment for experimental work.

With such accuracy, materials engineers can use simulation as a tool to develop new materials needed for specific use. As an example, a glass which is very resistant to hurricane can be simulated, tested and manufactured for real life use. The wind speeds against the strength of the material can be tested then manufactured.

Simple calculations can be done using normal desk top computers. When dealing with few atoms to study simple properties then even a lap top will work. But when the atoms are more than one hundred then the desk tops will delay or even crush because of the weight of the task. The study of molecules and molecular dynamics will demand more resources in terms of hours of usage. There are calculations that will run from days to weeks and will be impossible if these high speed machines were not accessible.

The good news is that if the internet connectivity is available, then HPC can be accessed if one has the user rights. CHPC has played a big role in this continent. Many students have benefited and are still benefiting from this facility. From the initial dream of enabling research and knowledge sharing, the center has become a force in organizing conferences and trainings in computation. This is a major boost to African continent which has gone down into enabling most students connect and work in collaborations which has led to quality publications. The beauty of HPC and in particular CHPC is its multidisciplinary. It is used by all science and engineering fields and at times one field can easily learn from a related field.

In this paper, the importance of High performance computing in computational research and how it has been used in Africa to foster materials exploration is discussed. It has been demonstrated by studying the structural properties of lanthanum fluoride $\left(\mathrm{LaF}_{3}\right)$. The study of properties of $\mathrm{LaF}_{3}$ has proven to be of interest for their many technological applications. These include their lubrification properties, their use in oxygen sensors and their efficiency as scintilators, laser sources, and detectors and in telecommunications applications [6].

The study entails the use of plane wave pseudo-potential (PW-PP) calculations [7]. Pseudo-potentials were extracted from the Quantum-Espresso database. Quantum Espresso implements a variety of methods and algorithms aimed at a chemically realistic modeling of materials from the nanoscale upwards [8]. The calculations are based on the solution of the density-functional theory [6] problem, using plane waves (PWs) basis set and pseudopotentials to represent electron- 
ion interactions and the generalized gradient approximations to study the electronic and structural properties of cubic and tysonite $\mathrm{LaF}_{3}$. It is known that ground state is the basis for understanding the fundamental properties of materials, which can provide much useful information for the applications of a material and for further studying its phase transition. We employed the norm conserving pseudopotentials to mimic the electron-ion interaction as discussed by Perdew and Zunger [9] within the local density approximation (LDA).

\section{Results}

Table 1. The calculated lattice parameters $a_{0}, c_{o}$ in Bohr and $c_{o} / a_{0}$ of $\mathrm{LaF}_{3}$ compared to experimental data.

\begin{tabular}{llllll}
\hline & $\mathbf{a}_{\mathbf{0}}$ & $\mathbf{c}_{\mathbf{0}}$ & (C/a) & Dev from Expt & Ref \\
\hline HPC & 7.245 & 7.376 & 1.02 & $0.3 \%$ & This work \\
EXPT & 7.200 & 7.376 & 1.024 & - & {$[10]$} \\
\hline
\end{tabular}

\section{Discussion}

Lattice parameter from this work deviates by $0.3 \%$ compared to experimental work. This indicates that with a proper pseudopotential one can do a computational work that is credible. Of course other properties of the material may not be accurate with one pseudopotential as with the others.

Other parameters such as the band gap of a material may not be as good as the lattice parameter. This has called for use of hybrid of pseudotentials to give results that are realistic.

\section{Conclusion and Recommendation}

From the results obtained, it has been established that computational methods are cheap, fast and accurate compared to the usual experimental and theoretical methods used in scientific research. It can also be noted that the results obtained from the HPC cluster agree well with the experimental results.

Needless to say, funding is a major challenge in scientific research in Africa. With no or little resources most researchers in Physics have opted for either theoretical or experimental Physics.

Experts in materials science are few and they are overstretched by the huge number of students that require their expertise. Most of these experts end up in teaching so many courses in the undergraduate living very little time for research.

Even with CHPC available to most of African countries, sensitization remains a challenge. Many countries have connectivity challenges. Institutions are poorly connected first to power and then to internet. If the internet is there, power outage is present and vice versa.

The other challenge is the open source softwares that may not meet the demand of the user. Some of these open source softwares may be slow. The softwares that are bought are very expensive and most of the higher learning institutions are either reluctant to purchase them or have priorities placed elsewhere.

Appreciations to Science Gateway in Africa (Sci-GaIA) group for allowing Quantum Espresso Code to be launched into their platform.

\section{References}

[1] Chpc http://www.top500.org

[2] Researchfundhttp://www.researchfund.go.ke

[3] Nacostihttp://www.nacosti.go.ke

[4] Young David Computational Chemistry:A practical Guide for Applying Techniques to Real world Problem.New York:John Willey \&sons pp 322-359 (2001)

[5] K. Lejaeghere et al, Reproducibility in density functionl theory calculations of solids.Science 381 (6280) aad 3000 (2016)

[6] F. Wang, X. G. Liu, Chem. Soc. Rev. 38(2009)976.

[7] S. Scandolo, P. Gannozzi, C. Cavazzoni, S. de Gironcoli, A. Pasquarello, and S. Baroni, Z. kristallogr, 220 pp. 574-579, (2005).

[8] P. Giannozzi, S. Baroni, et al., J. Phys.: Condens. Matter, 21, 395502 (2009)

[9] J. P. Perdew, A. Zunger, Phys. Rev. B 23 (10) (1981) 50485079

[10] A. Belzner, H. Schulz, G. Heger, Z. Kristallogr. 209(1994) 239. 